\title{
Does Sequence of Graft Tensioning Affect Outcomes in Combined Anterior and Posterior Cruciate Ligament Reconstructions?
}

\author{
Sung-Jae Kim MD, PhD, Sung-Hwan Kim MD, \\ Min Jung MD, Jong-Min Kim MD, Se-Won Lee MD
}

Received: 16 April 2014/ Accepted: 4 September 2014/Published online: 16 September 2014

(C) The Association of Bone and Joint Surgeons (B) 2014

\begin{abstract}
Background Controversy persists regarding the protocol for tensioning and securing the grafts in one-stage reconstruction of combined anterior cruciate ligament (ACL) and posterior cruciate ligament (PCL) injuries. Many authors have reported stability examinations and functional results after reconstruction for this relatively rare injury, and the best sequence for tensioning the grafts is not known.

Questions/purposes We sought to determine (1) if there are differences in postoperative anteroposterior stability in a protocol of simultaneous tensioning of both grafts and ACL-first fixation compared with a protocol of tensioning

Each author certifies that he or she, or a member of his or her immediate family, has no funding or commercial associations (eg, consultancies, stock ownership, equity interest, patent/licensing arrangements, etc) that might pose a conflict of interest in connection with the submitted article.

All ICMJE Conflict of Interest Forms for authors and Clinical Orthopaedics and Related Research ${ }^{\mathbb{R}}$ editors and board members are on file with the publication and can be viewed on request. Clinical Orthopaedics and Related Research ${ }^{\mathbb{R}}$ neither advocates nor endorses the use of any treatment, drug, or device. Readers are encouraged to always seek additional information, including FDA-approval status, of any drug or device prior to clinical use. Each author certifies that his or her institution approved the human protocol for this investigation and that all investigations were conducted in conformity with ethical principles of research.
\end{abstract}

S.-J. Kim, S.-H. Kim, M. Jung, S.-W. Lee ( $\square)$

Department of Orthopedic Surgery, Severance Hospital, Yonsei University College of Medicine, 250 Seongsanno,

Seodaemun-gu, Seoul 120-752, South Korea

e-mail: ssewon@naver.com

J.-M. Kim

Department of Orthopedic Surgery, Modu Hospital,

88 Nongogaero, Namdong-gu, Incheon 405-300, South Korea and fixation of the PCL first in one-stage reconstruction of combined ACL/PCL injuries; and (2) if there is a difference in postoperative functional outcome scores between the two protocols.

Methods Between 2001 and 2011, 29 patients underwent one-stage reconstruction of combined ACL and PCL injuries (the majority with medial collateral ligament [MCL] injuries, posterolateral corner [PLC] injuries, or both, in addition), of whom three patients (10\%) were lost to followup before 2 years, and one patient was excluded based on predefined criteria, leaving a total of 25 patients $(86 \%)$ for retrospective analysis in this report. Fourteen patients underwent one-stage reconstruction of these injuries with tensioning and fixation of the PCL graft first (PCL-first group), and 11 later patients underwent one-stage reconstruction of combined ACL/PCL injuries with simultaneous tensioning of both grafts and fixation of the ACL graft first (simultaneous-tensioning group). During the period in question, the technique used in the PCL-first group was used exclusively for these injuries between August 2001 and August 2008 and that used in the simultaneous-tensioning group was used between September 2008 and August 2011; there was no overlap between groups. The groups were similar in terms of demographics and length of followup. Each patient was assessed for associated injuries, preoperative and postoperative knee stability with anteroposterior stress radiographs, and was evaluated with the Lysholm knee score and International Knee Documentation Committee (IKDC) subjective and objective grading at the last followup after surgery.

Results At the last followup evaluation, patients treated with simultaneous tensioning and ACL-first fixation showed less instability on side-to-side difference of posterior stress radiography $(5 \pm 1 \mathrm{~mm}$ in the simultaneous-tensioning group versus $6 \pm 1 \mathrm{~mm}$ in the PCL-first group; effect size, 
$1.2 ; 95 \%$ confidence interval $[\mathrm{CI}], 0.5-2.3 ; \mathrm{p}=0.011)$, but with the numbers available, no difference on anterior stress radiography $(3 \pm 0.4 \mathrm{~mm}$ in the simultaneous-tensioning group versus $3 \pm 0.5 \mathrm{~mm}$ in PCL-first group; effect size, $0.4 ; 95 \% \mathrm{CI},-0.2$ to $0.5 ; \mathrm{p}=443)$. The simultaneous-tensioning group also had higher Lysholm knee scores $(87 \pm 5$ in the simultaneous-tensioning group versus $80 \pm 4$ in the PCL-first group; effect size, $1.8 ; 95 \% \mathrm{CI},-10.9$ to -2.7 ; $\mathrm{p}=0.001)$, IKDC subjective scores $(68 \pm 3$ in the simultaneous-tensioning group versus $58 \pm 3$ in the PCL-first group; effect size, $3.4 ; 95 \% \mathrm{CI},-14.2$ to $-8.6 ; \mathrm{p}<0.001$ ), and IKDC objective grades $(\mathrm{p}=0.037)$.

Conclusions In one-stage reconstruction of combined ACL and PCL injuries, a protocol of simultaneous tensioning both grafts and fixing the ACL graft first may be worth consideration. Arthroscopic reduction landmarks may prove helpful in this technique but require further validation.

Level of Evidence Level III, therapeutic study. See Guidelines for Authors for a complete description of levels of evidence.

\section{Introduction}

Single-stage reconstruction of both the anterior cruciate ligament (ACL) and posterior cruciate ligament (PCL) is an uncommon and challenging procedure. Although many authors recommend initial PCL graft fixation followed by ACL graft fixation [3-6, 21, 27, 33, 37], others support fixation of the ACL graft first [35]. Another proposed surgical option is simultaneous tensioning of the ACL and PCL grafts followed by initial PCL graft fixation [9, 21, 36].

The best protocol for the sequence of graft tensioning and graft fixation thus remains controversial [9, 23]. To our knowledge, there is no comparative study between different protocols.

We therefore sought to determine (1) if AP stability was improved with a protocol of simultaneous graft tensioning and ACL-first fixation compared with sequenced tensioning and fixation of the PCL first; and (2) if there was a difference in postoperative functional scores assessed with the Lysholm knee scoring scale [19] and the International Knee Documentation Committee (IKDC) subjective score and objective grading [10] between patients undergoing these two protocols.

\section{Patients and Methods}

We retrospectively reviewed the medical records of 29 patients (30 knees) who had undergone one-stage reconstruction of combined ACL and PCL injuries regardless of whether concomitant additional procedures were done for medial collateral ligament (MCL) and posterolateral corner
(PLC) injury between 2001 and 2011. All surgeries were performed by the senior author (S-JK). Patients with any of the following characteristics were excluded: (1) any prior surgery on the affected knee; (2) concomitant periarticular fracture; (3) instability of the contralateral knee; (4) malalignment of the lower extremity $(>5 \mathrm{~mm}$ of mechanical axis deviation as measured by standing hip-knee-ankle radiographs [26]); (5) Grade 3 or higher chondral lesion according to the Outerbridge grading system at arthroscopic examination [25]; (6) a severe meniscal injury requiring subtotal or total meniscectomy; and (7) duration of followup less than 24 months. Using these criteria, three patients (three knees [10\%]) were lost to followup before 2 years, and one patient (two knees) was excluded with bilateral injuries, leaving a total of 25 patients ( 25 knees [86\%]) included in the study.

\section{Participants/Study Subjects}

The PCL-first group consisted of 14 patients (14 knees) who had undergone one-stage combined ACL and PCL reconstruction with tensioning and fixation of the PCL first. The simultaneous-tensioning group consisted of 11 patients (11 knees) who were treated with one-stage combined ACL and PCL reconstruction with simultaneous tensioning of both grafts and fixation of the ACL first. During the period in question, the technique used in the PCL-first group was used exclusively for these injuries between August 2001 and August 2008 and that used in the simultaneous-tensioning group was used between September 2008 and August 2011; there was no overlap between groups. These groups were similar in terms of demographics and length of followup (Table 1). Specifically, sex distribution, mean age at surgery, time from injury to surgery distribution, and mean duration of followup did not differ significantly with the numbers available. The data of concomitant collateral ligament reconstruction in this study are shown (Table 1). Additional arthroscopic findings showed four cases of meniscal tears treated with partial meniscectomy, three cases of Outerbridge Grade 1 or 2 chondromalacia, and one case of a loose body in the PCL-first group. In the simultaneous-tensioning group, two cases of meniscal tears were treated with partial meniscectomy and two cases of Outerbridge Grade 1 or 2 chondromalacia were noted.

\section{Description of Surgery}

Anterolateral transtibial single-bundle PCL reconstruction with a one-incision technique using an allogenic Achilles tendon-bone graft [14] and a transtibial single-bundle ACL reconstruction technique using bone-patellar tendon-bone 
Table 1. Patient demographic data

\begin{tabular}{|c|c|c|c|}
\hline Demographic & $\begin{array}{l}\text { Group A } \\
(\mathrm{n}=14)\end{array}$ & $\begin{array}{l}\text { Group B } \\
(\mathrm{n}=11)\end{array}$ & $\mathrm{p}$ value \\
\hline Age (years), mean (range) & $30(20-51)$ & $34(20-48)$ & 0.270 \\
\hline Sex, male/female, number $(\%)$ & $10 / 4(71 / 29)$ & $9 / 2(82 / 18)$ & 0.554 \\
\hline $\begin{array}{l}\text { Affected side, right/left, } \\
\text { number }(\%)\end{array}$ & $8 / 6(57 / 43)$ & $7 / 4(64 / 36)$ & 0.312 \\
\hline $\begin{array}{l}\text { Duration from injury to surgery } \\
\text { (months) (mean, range) }\end{array}$ & $8(1-24)$ & $6(1-18)$ & 1.000 \\
\hline Acute $(<3$ weeks), number $(\%)$ & $2(14)$ & $2(18)$ & \\
\hline $\begin{array}{l}\text { Subacute or chronic } \\
\qquad>3 \text { weeks), number (\%) }\end{array}$ & $12(86)$ & $9(82)$ & \\
\hline $\begin{array}{l}\text { Duration of postoperative } \\
\text { followup (months), mean } \\
\text { (range) }\end{array}$ & $58(36-96)$ & $44(24-72)$ & 0.079 \\
\hline \multicolumn{3}{|c|}{ Ligamentous lesion requiring surgery, number $(\%)$} & 1.000 \\
\hline ACL, PCL & $1(7)$ & $1(9)$ & \\
\hline ACL, PCL, MCL & $4(29)$ & $3(27)$ & \\
\hline ACL, PCL, PLC & $5(36)$ & $4(36)$ & \\
\hline ACL, PCL, MCL, PLC & $4(29)$ & $3(27)$ & \\
\hline
\end{tabular}

$\overline{\mathrm{ACL}}=$ anterior cruciate ligament; $\mathrm{PCL}=$ posterior cruciate ligament; $\mathrm{MCL}=$ medial collateral ligament; $\mathrm{PLC}=$ posterolateral corner.

autograft $[13,15]$ were done in all enrolled patients in both groups. Our sequence of multiligament reconstruction is summarized (Fig. 1). After the fixation of the femoral side of the PCL and ACL grafts with the tibial side left unattached, if needed, anatomical PT and LCL reconstruction [16] using a tibialis posterior tendon allograft for PLC instability and a semitendinosus tenodesis [12] for combined medial instability were performed.

The two groups are then distinguished by the protocol for graft tensioning and fixation. In the PCL-first group, the PCL graft was tensioned and fixed first. The PCL graft was tensioned through 20 cyclic motion arcs to eliminate creep while manual tension was maintained on the tibial side. A slight anterior drawer force was applied while monitoring the tibial stepoff at $70^{\circ}$ of knee flexion. After the tibial stepoff was reduced normally, the PCL graft was secured on the tibial side with a bioabsorbable screw at $70^{\circ}$ of knee flexion. Twenty cyclic motions were repeated while the ACL graft was appropriately tensioned with manual posterior drawer force. After monitoring the tibial stepoff, the ACL graft was also fixed at $70^{\circ}$ of knee flexion [5].

In the simultaneous-tensioning group, the simultaneous tensioning of both grafts and fixation of the ACL graft were done first. Both grafts were tensioned simultaneously through 20 cyclic motion arcs with appropriate manual force. An arthroscopic reduction landmark (contact between the notch of the lateral femoral condyle and the anterior horn of the lateral meniscus) (Fig. 2A) was used and the ACL graft was secured with an absorbable interference screw with the knee in full extension. The cyclic motion arc of the knee was then repeated 20 times while maintaining maximal manual tension on the PCL graft. A second arthroscopic reduction landmark (contact between the medial femoral condyle and the posterior horn of the medial meniscus) (Fig. 2B) was used, and the PCL graft was secured with an interference screw at $70^{\circ}$ to $90^{\circ}$ of knee flexion.

\section{Postoperative Rehabilitation}

All patients in both groups followed an identical postoperative rehabilitation protocol described by Edson et al. [2] The involved knee was immobilized in extension with a hinged knee brace for the first 4 weeks. Quadriceps strengthening and mobilization with patellar exercises were initiated immediately after surgery. Toe-touch weightbearing was allowed. For 4 to 12 weeks, weightbearing with crutches as tolerated and limited flexion of the knee with a gradual increase up to $90^{\circ}$ were encouraged. After 12 weeks, the brace was removed and stationary bicycling, stairclimbing, full flexion, and squatting were gradually allowed. At 4 to 6 months, swimming and cycling were permitted. Return to sports activity involving jumping and pivoting was permitted at 9 to 12 months after surgery.

\section{Outcome Measures}

Study outcomes of interest were assessed at baseline (before surgery) and at the last followup after surgery. All manual examinations were performed by the senior author (S-JK) and trained orthopaedic surgeons. Radiological evaluation by stress radiographs was performed by two different orthopaedic surgeons (J-HL, H-SK), who were uninformed of the study objective, and the mean of these two numerical values was used. Laxity of the ACL and PCL was evaluated with a Telos stress device (Telos, Marburg, Germany) with a load of $150 \mathrm{~N}$ at $20^{\circ}$ and $90^{\circ}$ of flexion, respectively. Values of the affected knee were compared with those of the normal contralateral knee. The bony landmarks and method to measure the magnitude of translation described in previous reports were used [20, 30, 32]. One horizontal line tangential to the tibial plateau was drawn. Two additional vertical lines perpendicular to this horizontal line were drawn. Each line passed the midpoint between the vertical lines tangential to the most posterior outlines of both femoral condyles and both tibial plateaus, respectively. The distance between these two vertical lines was measured to determine the skeletal anterior or posterior translation. The stress radiographs were evaluated through digital radiography (Centricity PACS; GE Medical System Information Technologies, Milwaukee, WI, USA). The values of the affected knee were compared with 


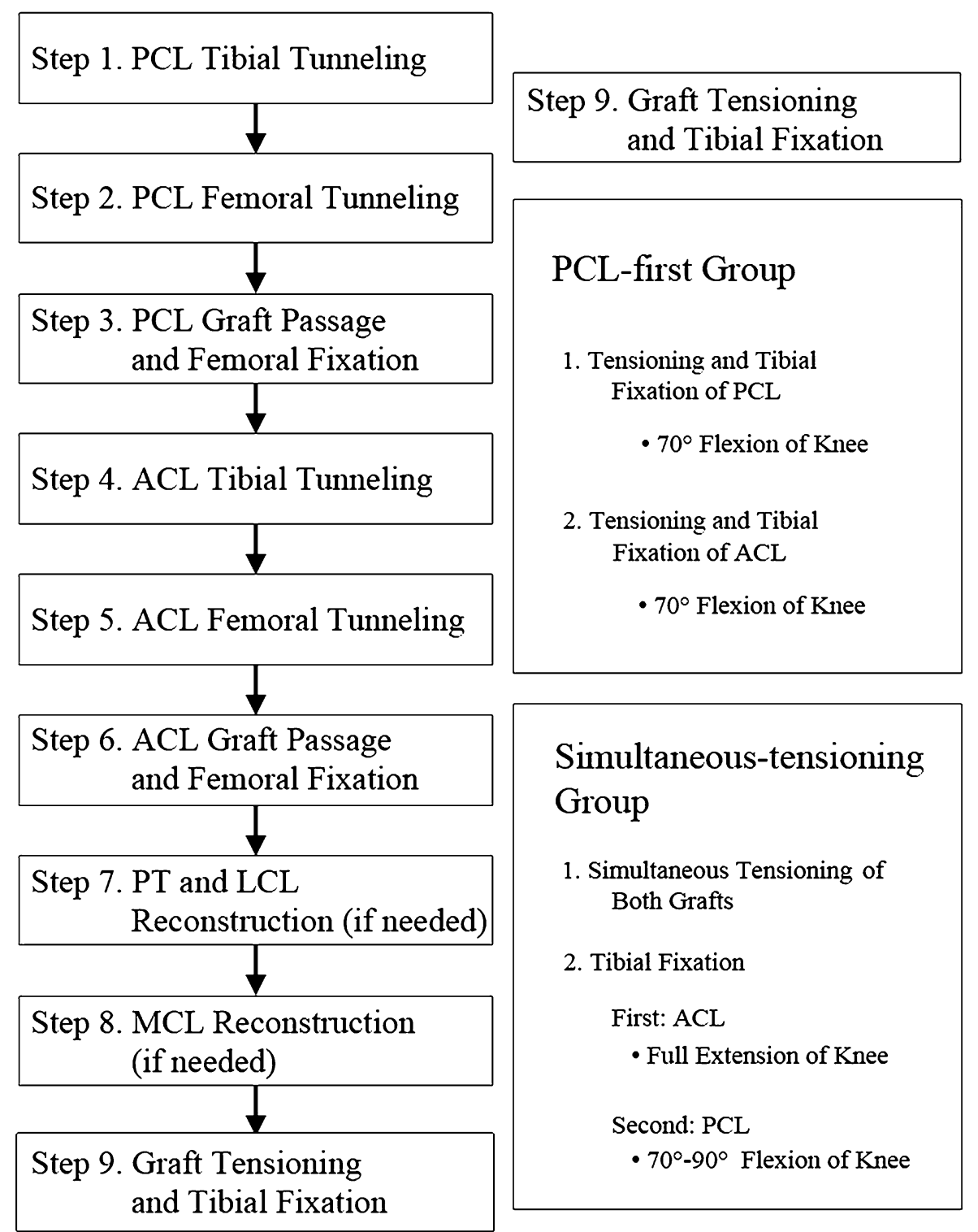

Fig. 1 The sequence of multiligament reconstruction is shown.

those of the normal contralateral knee for side-to-side differences (SSDs). Preoperative and postoperative functional outcomes were assessed with the Lysholm knee score [19] and the IKDC subjective score and examination form [10].

\section{Statistical Analysis, Study Size}

Comparison between the two groups in preoperative and postoperative data was performed with a nonparametric test (Wilcoxon signed-rank test) for continuous numerical data and the Fisher's exact test for categorical data. The interobserver reliability in measuring the radiological variables was evaluated with intraclass correlation coefficients (ICCs) with $95 \%$ confidence intervals (CIs).
Statistical analysis was performed using SPSS software (Version 19; SPSS Inc, Chicago, IL, USA). The level of significance was set at $\mathrm{p}<0.05$.

The minimum clinically important difference (MCID) of AP stress radiography using the Telos device is not known. The MCID of the Lysholm score has been estimated to be 10 points and the IKDC score to be 12 points $[1,11]$.

\section{Results}

Translation on Stress Radiography

There was no difference between the two groups with the numbers available on posterior stress radiography 


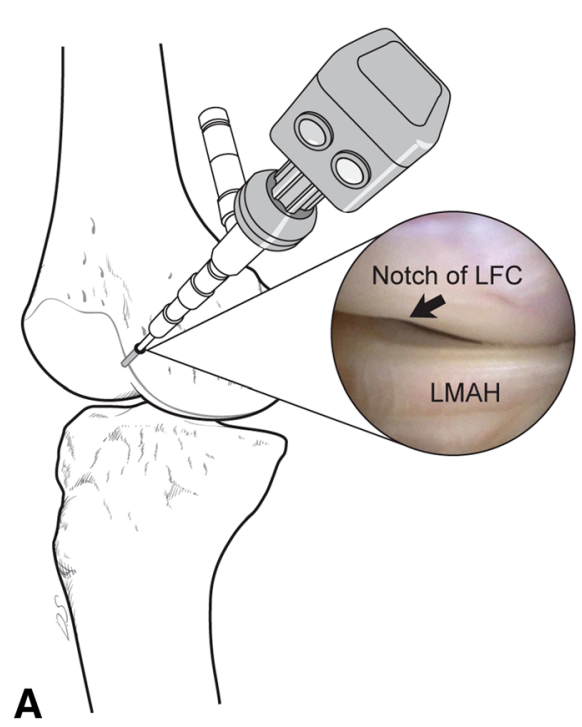

Fig. 2A-B In the simultaneous-tensioning group, the protocol of simultaneous tensioning and fixing the ACL graft first was used after the fixation of the femoral side of the PCL and ACL grafts. Both grafts were tensioned simultaneously through 20 time cyclic motions. With the knee in full extension, the ACL graft was secured with an absorbable interference screw after arthroscopic reduction landmark (A) in which the notch of lateral femoral condyle (Notch of LFC) engaged on the lateral meniscus anterior horn (LMAH) was observed.

preoperatively $(11 \pm 1 \mathrm{~mm}$ in the PCL-first group and $12 \pm 1 \mathrm{~mm}$ in the simultaneous-tensioning group; effect size, $0.3 ; 95 \% \mathrm{CI},-1.1$ to $0.6 ; \mathrm{p}=0.381$ ) (Table 2). The simultaneous-tensioning group showed less postoperative instability on side-to-side posterior stress radiography compared with the PCL-first group $(5 \pm 1 \mathrm{~mm}$ in the simultaneous-tensioning group versus $6 \pm 1 \mathrm{~mm}$ in the PCL-first group; effect size, 1.2; 95\% CI, 0.5-2.3; $\mathrm{p}=0.011$ ) (Table 3) at the last followup evaluation. However, these differences were small and of questionable clinical importance.

Anterior translation on stress radiography showed no difference between two groups with the numbers available both preoperatively $(8 \pm 1 \mathrm{~mm}$ in PCL-first group and $8 \pm 1 \mathrm{~mm}$ in simultaneous-tensioning group; effect size, $0.5 ; 95 \% \mathrm{CI},-1.1$ to $0.2 ; \mathrm{p}=0.162$ ) (Table 2 ) and postoperatively $(3 \pm 0.5 \mathrm{~mm}$ in the PCL-first group and $3 \pm 0.4 \mathrm{~mm}$ in the simultaneous-tensioning group; effect size, $0.4 ; 95 \% \mathrm{CI},-0.2$ to $0.5 ; \mathrm{p}=443$ ) (Table 3 ). The reliability was good in terms of interpreting the radiographic measurements (ICC $=0.956$ in the PCL-first group and ICC $=0.959$ in the simultaneous-tensioning group).

\section{Knee Scores}

With the available numbers, there was no difference between both groups with regard to preoperative Lysholm knee scores,

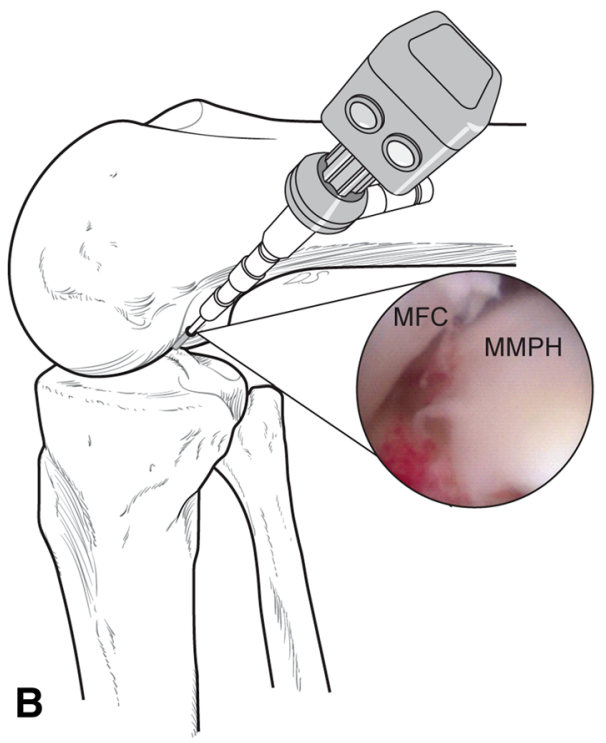

After tensioning of the PCL graft by an additional 20 time cyclic motions, another arthroscopic reduction landmark in which the medial femoral condyle (MFC) contacts with the medial meniscus posterior horn (MMPH) (B) can be observed through the posteromedial portal in $70^{\circ}$ to $90^{\circ}$ flexion of the knee. Figures created by Dong-Su Jang, Medical Illustrator, Medical Research Support Section, Yonsei University College of Medicine, Seoul, Korea. Published with permission.

IKDC subjective scores, and IKDC objective grade (Table 2). However, the postoperative outcomes as measured by Lysholm knee scores $(87 \pm 5$ in the simultaneous-tensioning group versus $80 \pm 4$ in the PCL-first group; effect size, 1.8; $95 \% \mathrm{CI},-10.9$ to $-2.7 ; \mathrm{p}=0.001$ ), IKDC subjective scores (68 \pm 3 in simultaneous-tensioning group versus $58 \pm 3$ in PCL-first group; effect size, $3.4 ; 95 \% \mathrm{CI},-14.2$ to -8.6 ; $\mathrm{p}<0.001)$, and IKDC objective grades $(\mathrm{p}=0.037)$ of the simultaneous-tensioning group are favorable compared with those of the PCL-first group (Table 3). There are similar results regarding amount of difference before and after surgery between groups (Table 4). However, in many cases these differences were smaller than the MCID and so may not have been perceptible to the typical patient.

\section{Discussion}

Several graft-tensioning and fixation protocols have been reported in the setting of one-stage reconstruction of combined ACL and PCL injuries: tensioning and fixation of the PCL graft first [3-6, 8, 27, 33, 37], simultaneous tensioning of both grafts and fixation of the PCL first $[9,21,36]$, and tensioning and fixation of the ACL graft first [35]. In such combined reconstructive procedures, there has been general consensus about the tendency for posterior 
Table 2. Comparison of preoperative variables between groups

\begin{tabular}{lcll}
\hline Preoperative variable & $\begin{array}{c}\text { Group A } \\
(\mathrm{n}=14)\end{array}$ & $\begin{array}{l}\text { Group B } \\
(\mathrm{n}=11)\end{array}$ & p value \\
\hline $\begin{array}{l}\text { SSD in posterior } \\
\text { translation* (mm) }\end{array}$ & $11 \pm 1$ & $12 \pm 1$ & 0.381 \\
$\begin{array}{l}\text { SSD in anterior translation* } \\
\quad(\mathrm{mm})\end{array}$ & $8 \pm 1$ & $8 \pm 1$ & 0.162 \\
Lysholm knee score* & $58 \pm 5$ & $58 \pm 4$ & 0.640 \\
IKDC subjective score* & $39 \pm 4$ & $40 \pm 4$ & 0.377 \\
Symptom & $17 \pm 2$ & $17 \pm 3$ & 0.572 \\
Sports & $15 \pm 3$ & $15 \pm 2$ & 0.727 \\
Function & $3 \pm 1$ & $3 \pm 1$ & 0.434 \\
IKDC objective grade, number $(\%)^{\dagger}$ & & 0.999 \\
A & 0 & 0 & \\
B & 0 & 0 & \\
C & $2(14)$ & $2(18)$ & \\
D & $12(86)$ & $9(82)$ & \\
\hline
\end{tabular}

* The values are given as mean $\pm \mathrm{SD}$; the values are given as number $(\%) ; \mathrm{SSD}=$ side-to-side difference; IKDC = International Knee Documentation Committee.

Table 3. Comparison of postoperative variables between groups

\begin{tabular}{lllr}
\hline Postoperative variable & $\begin{array}{l}\text { Group A } \\
(\mathrm{n}=14)\end{array}$ & $\begin{array}{l}\text { Group B } \\
(\mathrm{n}=11)\end{array}$ & p value \\
\hline $\begin{array}{l}\text { SSD in posterior } \\
\text { translation* (mm) }\end{array}$ & $6 \pm 1$ & $5 \pm 1$ & 0.011 \\
$\begin{array}{l}\text { SSD in anterior translation* } \\
\quad(\mathrm{mm})\end{array}$ & $3 \pm 0.5$ & $3 \pm 0.4$ & 0.443 \\
Lysholm knee score* & $80 \pm 4$ & $87 \pm 5$ & 0.001 \\
IKDC subjective score* & $58 \pm 3$ & $68 \pm 3$ & $<0.001$ \\
Symptom & $27 \pm 2$ & $30 \pm 2$ & $<0.001$ \\
Sports & $26 \pm 2$ & $31 \pm 2$ & $<0.001$ \\
Function & $6 \pm 1$ & $7 \pm 1$ & $<0.001$ \\
IKDC objective grade, number $(\%)$ & & 0.037 \\
A & $0(0)$ & $3(27)$ & \\
B & $5(36)$ & $6(55)$ & \\
C & $7(50)$ & $1(9)$ & \\
D & $2(14)$ & $1(9)$ & \\
\hline
\end{tabular}

* The values are given as mean $\pm \mathrm{SD} ; \mathrm{SSD}=$ side-to-side difference; IKDC = International Knee Documentation Committee.

instability $[31,36]$, the superior predictability of the ACL reconstructive procedure compared with the PCL reconstructive procedure $[6,24,33,36]$, and the importance of PCL function restoration to results $[4,23,36]$. We had previously used a protocol similar to that introduced by Fanelli et al. [5] in the earlier PCL-first group of this study but found the outcomes were unsatisfactory with regard to posterior stability. We therefore revised the protocol with the sequence of simultaneous tensioning both grafts and ACL graft fixation first and compared the different
Table 4. Comparison of amount of difference before and after surgery between groups

\begin{tabular}{lccr}
\hline Pre-/postoperative variable & $\begin{array}{l}\text { Group A } \\
(\mathrm{n}=14)\end{array}$ & $\begin{array}{c}\text { Group B } \\
(\mathrm{n}=11)\end{array}$ & p value \\
\hline $\begin{array}{l}\text { SSD in posterior } \\
\text { translation (mm) }\end{array}$ & $-5 \pm 2$ & $-7 \pm 1$ & 0.020 \\
$\begin{array}{l}\text { SSD in anterior translation } \\
\quad(\mathrm{mm})\end{array}$ & $-4 \pm 1$ & $-5 \pm 1$ & 0.999 \\
Lysholm knee score & $22 \pm 5$ & $29 \pm 5$ & 0.003 \\
IKDC subjective score & $28 \pm 4$ & $38 \pm 6$ & $<0.001$ \\
$\quad$ Symptom (37) & $10 \pm 2$ & $13 \pm 3$ & 0.010 \\
Sports (40) & $11 \pm 3$ & $16 \pm 2$ & 0.002 \\
Function (10) & $3 \pm 1$ & $5 \pm 1$ & $<0.001$ \\
\hline
\end{tabular}

The values are given as mean $\pm \mathrm{SD} ; \mathrm{SSD}=$ side-to-side difference; IKDC = International Knee Documentation Committee.

approaches in terms of side-to-side stability and validated knee functional scores.

There were several limitations in our study. First, the study was based on retrospective review and contained a relatively small cohort of patients, because reconstruction of combined ACL and PCL injuries is relatively uncommon. Second, the assignment of patients was not random but sequential. The sequential assignment could potentially entail confounding variables and possible cotreatment bias as a result of other changes over time in terms of physical therapy, pain management, surgical instrumentation, etc. Additionally, there is a learning curve in performing onestage reconstruction of combined ACL and PCL injuries like most complex operative procedures, but our senior author (S-JK) had more than 10 years of accumulated experience in this procedure before the first case in this study. Third, most patients who had undergone reconstruction of combined ACL and PCL injuries also had concomitant collateral ligament reconstruction in this study. Although collateral ligamentous instabilities can affect the postoperative anterior and posterior stability, no differences were detected in terms of stability on postoperative varus and valgus stress radiography between the two groups with the numbers available. There was only one patient in each group with isolated ACL/ PCL injury without concomitant ligamentous injuries so this group was too small to serve as a control. Fourth, although the postoperative outcomes of the simultaneous-tensioning group were favorable, the maximal differences in functional scores were not substantial, and the difference in Lysholm scores and IKDC scores was less than the reported MCID. Fifth, radiological evaluation by stress radiography used in this study to examine the anterior and posterior translation is inevitably subjective. Measurements were performed twice by different orthopaedic surgeons to lower subjectivity, introducing a potential issue of interobserver repeatability, but the agreement between the observers was very high. 
Many articles have studied one-stage reconstruction of combined ACL/PCL injuries [3-6, 8, 9, 17, 21, 23, 24, 27, $31,33,35-37]$. In a biomechanical study published in 2003, Markolf et al. [23] demonstrated tensioning the PCL graft first improves AP stability when tensioning both grafts at the same flexion angle. They demonstrated that a $30^{\circ}$ flexion angle would be more suitable rather than $90^{\circ}$ when both grafts are tensioned at the same flexion angle. Fanelli et al. [3-6, 18] used a graft tensioning protocol where tensioning and fixing the PCL graft were followed by tensioning and fixing the ACL graft at the same $70^{\circ}$ flexion angle. They believed tensioning the ACL graft at $70^{\circ}$ flexion enables surgeons to maintain the neutral position of the knee by monitoring tibial stepoff. Postoperative posterior stress radiographs with the KT-1000 arthrometer of their 35 cases showed a SSD with normal contralateral knees of 0 to $3 \mathrm{~mm}$ in $52 \%$ of the knees, 4 to $5 \mathrm{~mm}$ in $24 \%$, and 6 to $10 \mathrm{~mm}$ in 19\%. Wascher et al. [36] described a protocol with simultaneous tensioning and fixing the PCL graft first in a 13-case series of one-stage reconstruction of ACL and PCL injuries. Both grafts were tensioned simultaneously with the knee flexed to $90^{\circ}$ under while checking that normal tibiofemoral stepoff was reproduced. The PCL graft was tensioned with approximately $45 \mathrm{~N}$ of force and fixed at $90^{\circ}$ and then the ACL graft was tensioned and fixed at $20^{\circ}$ flexion of the knee with same force. Their mean value of AP SSDs was reported to be $5 \mathrm{~mm}$ at $20^{\circ}$ of knee flexion and $5 \mathrm{~mm}$ at $70^{\circ}$ of flexion. Mariani et al. [21] retrospectively evaluated 15 patients who underwent arthroscopically assisted simultaneous ACL and PCL reconstruction with hamstring and patellar tendon autograft, respectively. Their protocol used simultaneous tensioning and fixation of both grafts at $30^{\circ}$ of knee flexion and also used arthroscopic landmarks for achieving the appropriate tibiofemoral relationship. With the knee held in $30^{\circ}$ of knee flexion, the PCL and ACL grafts were fixed to the tibia. They noted $3 \mathrm{~mm}$ of mean SSD for anterior stress and $6 \mathrm{~mm} / 7 \mathrm{~mm}$ mean SSD for posterior stress referenced from the lateral/medial tibial plateau. In the present study, when the grafts were tensioned simultaneously and the ACL graft was fixed first, with numbers available, we did not detect a difference in anterior translation on stress radiography but found reduced posterior translation on stress radiography compared with patients treated with a PCL-first approach. However, the observed differences were small, on the order of $1 \mathrm{~mm}$ or so of side-to-side difference. This may well be smaller than the MCID and so may not be perceptible to the typical patient. Still, we believe our work suggests that the outcomes for posterior translation of simultaneous tensioning and fixing the ACL first are not inferior in contrast to the existing concept that the PCL graft should be fixed first [3, 5, 23]. Likewise, fixing the ACL first in our study did not negatively affect anterior translation in contrary to an general expectation [24] that the ACL graft would to be slack or inadequately tensioned when the ACL graft was fixed first [23].

The mechanical stability of the PCL-first group in our study using the protocol of Fanelli et al. [5] was inferior to that of other investigators, including Fanelli, but the mechanical stability of the simultaneous-tensioning group was comparable $[5,21,27,33,36]$. There may be numerous explanations for the inferior results in the PCLfirst group, but we believe the favorable outcomes of the simultaneous-tensioning group may support the hypothetical basis of the protocol with simultaneous tensioning of both grafts and fixing the ACL graft first, as follows: the full extension position is optimal to set the tibiofemoral articulation in a reduced position on the basis of existence of the arthroscopic landmark of the terminal sulcus of the lateral femoral condyle ("notch"), contacting the anterior horn of the lateral meniscus. Additionally the AP translation is also restrained by the tensed posterior capsule and the collateral ligaments. Even if the collateral ligaments are injured, they are still expected to function as a restraint because fixation of collateral ligaments is performed before the final fixation of the bicruciate grafts (Fig. 1). When the PCL graft is tensioned at $70^{\circ}$ to $90^{\circ}$ of knee flexion, the tensioning of the PCL graft may be slightly overtensioned because its tension is not compromised after fixation of the ACL graft. This slight overtensioning of the PCL graft might be helpful to avoid posterior laxity. In addition, tensioning the PCL graft simultaneously and fixing it after the ACL graft might obviate the concern that the later tensioning of the ACL graft could disrupt the tibial stepoff achieved in the sequence of tensioning PCL first. However, further study should be performed to test this hypothesis.

When the grafts were tensioned simultaneously and the ACL was fixed first, with numbers available, we found higher functional scores compared with patients treated with a PCL-first approach. Compared with the results in several studies that recorded postoperative outcome by the scoring scales used in this study, the results of the simultaneoustensioning group were quite high in the Lysholm score, ranging from 77 to $92[5,7,18,22,28,29,34,36]$ in the literature; outstanding in "excellent/good: percentage of IKDC objective grading; ranged from 24 to 92 [3, 8, 18, 22, $28,29,33,34,36,37]$ but slightly below in the subjective score of IKDC, ranging from 61 to 92 [8, 33, 37]. In our investigation, poor IKDC subjective scores likely reflected little interest in returning to sports activities secondary to fear of reinjury. In addition, a low proportion of sport injuries (traffic accident: $65 \%$, fall down/slippage: $15 \%$, sport injuries: $19 \%$ ) in this study perhaps contributed to the low IKDC subjective score related to sports activity. However, the difference we found in functional scores between the two groups of our study was smaller than the MCID for those 
validated knee scores. Even so, we believe the difference is notable between two groups without outliers and in the fact that the simultaneous-tensioning group showed a high percentage of "excellent/good" in IKDC objective form.

This protocol of simultaneous tensioning both ACL and PCL grafts with ACL-first fixation should receive consideration when performing one-stage reconstruction of combined ACL and PCL injuries. Our modification of the technique described by Mariani et al. [21] for arthroscopic landmarks may allow for more accurate reduction than fluoroscopic or manual palpation methods but will require further validation. Future studies should focus on refining these techniques, validating arthroscopic reduction landmarks, and defining the stability parameters that improve functional results.

Acknowledgments We thank Dong-Su Jang (medical illustrator, Medical Research Support Section, Yonsei University College of Medicine, Seoul, Korea) for his support with the medical figure and Jae-Hoo Lee MD, and Hak-Soo Kim MD (clinical fellow, Department of Orthopaedic Surgery, Severance Hospital, Yonsei University College of Medicine, Seoul, Korea) for their support with radiological data collection.

\section{References}

1. Briggs KK, Kocher MS, Rodkey WG, Steadman JR. Reliability, validity, and responsiveness of the Lysholm knee score and Tegner activity scale for patients with meniscal injury of the knee. J Bone Joint Surg Am. 2006;88:698-705.

2. Edson CJ, Fanelli GC, Beck JD. Rehabilitation after multipleligament reconstruction of the knee. Sports Med Arthrosc. 2011;19:162-166.

3. Fanelli GC, Edson CJ. Arthroscopically assisted combined anterior and posterior cruciate ligament reconstruction in the multiple ligament injured knee: 2- to 10-year follow-up. Arthroscopy. 2002;18:703-714.

4. Fanelli GC, Edson CJ, Reinheimer KN. Evaluation and treatment of the multiligament-injured knee. Instr Course Lect. 2009;58:389-395.

5. Fanelli GC, Giannotti BF, Edson CJ. Arthroscopically assisted combined anterior and posterior cruciate ligament reconstruction. Arthroscopy. 1996;12:5-14.

6. Fanelli GC, Tomaszewski DJ. Allograft use in the treatment of the multiple ligament injured knee. Sports Med Arthrosc. 2007;15:139-148.

7. Harner CD, Waltrip RL, Bennett CH, Francis KA, Cole B, Irrgang JJ. Surgical management of knee dislocations. J Bone Joint Surg Am. 2004;86:262-273.

8. Hart JM, Blanchard BF, Hart JA, Montgomery SC, Schoderbek $\mathrm{R}$, Miller MD. Multiple ligament knee reconstruction clinical follow-up and gait analysis. Knee Surg Sports Traumatol Arthrosc. 2009;17:277-285.

9. Hayashi R, Kitamura N, Kondo E, Anaguchi Y, Tohyama H, Yasuda K. Simultaneous anterior and posterior cruciate ligament reconstruction in chronic knee instabilities: surgical concepts and clinical outcome. Knee Surg Sports Traumatol Arthrosc. 2008;16:763-769.

10. Hefti F, Muller W, Jakob RP, Staubli HU. Evaluation of knee ligament injuries with the IKDC form. Knee Surg Sports Traumatol Arthrosc. 1993;1:226-234.
11. Irrgang JJ, Anderson AF, Boland AL, Harner CD, Neyret $\mathrm{P}$, Richmond JC, Shelbourne KD, International Knee Documentation Committee. Responsiveness of the International Knee Documentation Committee Subjective Knee Form. Am J Sports Med. 2006;34:1567-1573.

12. Kim SJ, Choi NH, Shin SJ. Semitendinosus tenodesis for medial instability of the knee. Arthroscopy. 2001;17:660-663.

13. Kim SJ, Jo SB, Kumar P, Oh KS. Comparison of single- and double-bundle anterior cruciate ligament reconstruction using quadriceps tendon-bone autografts. Arthroscopy. 2009;25:70-77.

14. Kim SJ, Kim HK, Kim HJ. A modified endoscopic technique for posterior cruciate ligament reconstruction using allograft. Arthroscopy. 1998;14:643-648.

15. Kim SJ, Moon HK, Kim SG, Chun YM, Oh KS. Does severity or specific joint laxity influence clinical outcomes of anterior cruciate ligament reconstruction? Clin Orthop Relat Res. 2010;468:1136-1141.

16. Kim SJ, Park IS, Cheon YM, Ryu SW. New technique for chronic posterolateral instability of the knee: posterolateral reconstruction using the tibialis posterior tendon allograft. Arthroscopy. 2004;20(Suppl 2):195-200.

17. Levy BA, Dajani KA, Whelan DB, Stannard JP, Fanelli GC, Stuart MJ, Boyd JL, MacDonald PA, Marx RG. Decision making in the multiligament-injured knee: an evidence-based systematic review. Arthroscopy. 2009;25:430-438.

18. Liow RY, McNicholas MJ, Keating JF, Nutton RW. Ligament repair and reconstruction in traumatic dislocation of the knee. J Bone Joint Surg Br. 2003;85:845-851.

19. Lysholm J, Gillquist J. Evaluation of knee ligament surgery results with special emphasis on use of a scoring scale. Am J Sports Med. 1982;10:150-154.

20. Margheritini F, Mancini L, Mauro CS, Mariani PP. Stress radiography for quantifying posterior cruciate ligament deficiency. Arthroscopy. 2003;19:706-711.

21. Mariani PP, Margheritini F, Camillieri G. One-stage arthroscopically assisted anterior and posterior cruciate ligament reconstruction. Arthroscopy. 2001;17:700-707.

22. Mariani PP, Santoriello P, Iannone S, Condello V, Adriani E. Comparison of surgical treatments for knee dislocation. Am J Knee Surg. 1999;12:214-221.

23. Markolf KL, O'Neill G, Jackson SR, McAllister DR. Reconstruction of knees with combined cruciate deficiencies: a biomechanical study. J Bone Joint Surg Am. 2003;85:1768-1774.

24. Noyes FR, Barber-Westin SD. Reconstruction of the anterior and posterior cruciate ligaments after knee dislocation. Use of early protected postoperative motion to decrease arthrofibrosis. Am J Sports Med. 1997;25:769-778.

25. Outerbridge RE. The etiology of chondromalacia patellae. J Bone Joint Surg Br. 1961;43:752-757.

26. Paley D, Herzenberg JE, Tetsworth K, McKie J, Bhave A. Deformity planning for frontal and sagittal plane corrective osteotomies. Orthop Clin North Am. 1994;25:425-465.

27. Piontek T, Ciemniewska-Gorzela K, Szulc A, Naczk J, Wardak M, Trzaska T, Dudzinski W, Grygorowicz M. Arthroscopically assisted combined anterior and posterior cruciate ligament reconstruction with autologous hamstring grafts-isokinetic assessment with control group. PloS One. 2013;8:e82462.

28. Richter M, Bosch U, Wippermann B, Hofmann A, Krettek C. Comparison of surgical repair or reconstruction of the cruciate ligaments versus nonsurgical treatment in patients with traumatic knee dislocations. Am J Sports Med. 2002;30:718-727.

29. Rios A, Villa A, Fahandezh H, de Jose C, Vaquero J. Results after treatment of traumatic knee dislocations: a report of 26 cases. $J$ Trauma. 2003;55:489-494. 
30. Schulz MS, Russe K, Lampakis G, Strobel MJ. Reliability of stress radiography for evaluation of posterior knee laxity. Am J Sports Med. 2005;33:502-506.

31. Shelbourne KD, Porter DA, Clingman JA, McCarroll JR, Rettig AC. Low-velocity knee dislocation. Orthop Rev. 1991;20:995-1004.

32. Staubli HU, Jakob RP. Posterior instability of the knee near extension. A clinical and stress radiographic analysis of acute injuries of the posterior cruciate ligament. J Bone Joint Surg Br. 1990;72:225-230.

33. Strobel MJ, Schulz MS, Petersen WJ, Eichhorn HJ. Combined anterior cruciate ligament, posterior cruciate ligament, and posterolateral corner reconstruction with autogenous hamstring grafts in chronic instabilities. Arthroscopy. 2006;22: 182-192.
34. Tzurbakis M, Diamantopoulos A, Xenakis T, Georgoulis A. Surgical treatment of multiple knee ligament injuries in 44 patients: 2-8 years follow-up results. Knee Surg Sports Traumatol Arthrosc. 2006;14:739-749.

35. Van Tongel A, MacDonald PB. How I manage the multipleligament injured knee. Operative Techniques in Sports Medicine. 2010;18:245-249.

36. Wascher DC, Becker JR, Dexter JG, Blevins FT. Reconstruction of the anterior and posterior cruciate ligaments after knee dislocation. Results using fresh-frozen nonirradiated allografts. Am J Sports Med. 1999;27:189-196.

37. Zhao J, He Y, Wang J. Simultaneous arthroscopic reconstruction of the anterior and posterior cruciate ligaments with autogenous hamstring tendons. Arthroscopy. 2006;22:497-504. 